\title{
Green's functions for boundary value problems of generalized fractional differential equations with p-Laplacian
}

\author{
Arjumand Seemab*(D), Mujeeb ur Rehman (10) \\ School of Natural Sciences, \\ National University of Sciences and Technology, Sector H-12 Islamabad Pakistan
}

\begin{abstract}
We utilize the recently presented generalized fractional derivatives, which are not the same as standard Caputo and Riemann-Liouville fractional derivatives, to reformulate some boundary value problems of fractional differential equations. For some classes of generalized fractional differential equations with boundary conditions build up, we find the corresponding Green's functions and establish their properties under suitable assumptions and we also demonstrate the applicability of these properties of the Green's functions to establish some existence results via fixed point theorems.
\end{abstract}

Mathematics Subject Classification (2010). 26A33, 34B18, 34B27

Keywords. generalized fractional derivatives, positive solutions, p-Laplacian, Green's function, fixed-point theorem

\section{Introduction}

Fractional differential equations have attracted extensive interest at recent times. It is due to the intensive development of the theory of fractional calculus and because of the utilizations of such developments in the various fields of sciences and engineering [1-3, 5-9, 12, 15, 21, 23-25, 27].

Amid the previous decades, many considerations have been centered around the investigation of conditions with p-Laplacian differential operator. The inspiration for those works comes from the applications of different physical and natural phenomena. The researchers tried to suggest several types of fractional operators to describe more accurately these phenomena [4]. The Caputo-Katugampola fractional operator generalizes the classical Caputo fractional operator. Models based on generalized fractional derivatives may be more accurate than the models based on classical fractional derivatives. There exist a substantial number of papers committed to the existence of solutions for the equations with p-Laplacian operators $[10,20]$.

Much attention is paid to the investigation of the existence and multiplicity of positive solutions for boundary value problems of fractional differential equations by applying

\footnotetext{
*Corresponding Author.

Email addresses: arjumandseemab52@gmail.com (A. Seemab), mujeeburrrehman345@yahoo.com (M. Rehman)

Received: 30.08.2018; Accepted: 28.09.2019
} 
different techniques from nonlinear analysis (fixed-point theorems [16, 26, 29, 31], upper and lower solutions method [11,22], fixed-point index theory [32], etc.).

The boundary value problems of fractional differential equations with p-Laplacian have been studied in $[13,28]$. However to the best of our knowledge, no work has been found in the literature on the existence of multiple positive solutions for the generalized fractional boundary value problems with p-Laplacian. Motivated by some results in $[17,18]$, in this article, with the fractional differential equations and boundary conditions established, we find the corresponding Green's functions and prove their positivity under appropriate assumptions. We also demonstrate the applications of the properties of Green's functions by making the use of fixed point theorems.

The sections of this article are organized as follows. In Section 2, we present some definitions and lemmas. In Section 3 and 4, we derive the Green's functions of Caputo type and Riemann-Liouville type boundary value problems with generalized fractional derivatives and establish some useful properties. Applications are given in Section 5.

\section{Preliminaries}

In this section, we give some background materials from the the theory of fractional calculus to facilitate analysis of the boundary value problems.

Definition 2.1 ([19]). The general left-sided fractional integral

$$
{ }^{\rho} I_{a^{+}}^{\alpha} f(x)=\frac{\rho^{1-\alpha}}{\Gamma(\alpha)} \int_{a}^{x} \frac{\tau^{\rho-1} f(\tau)}{\left(x^{\rho}-\tau^{\rho}\right)^{1-\alpha}} d \tau
$$

for $x>a, \rho>0$, if the integral exists.

The generalized fractional derivative [19], corresponding to the generalized fractional integral (2.1), is defined for $0 \leq a<x$, by

$$
{ }^{\rho} D_{a^{+}}^{\alpha} f(x)=\frac{\rho^{\alpha-n+1}}{\Gamma(n-\alpha)}\left(x^{1-\rho} \frac{d}{d x}\right)^{n} \int_{a}^{x} \frac{\tau^{\rho-1} f(\tau)}{\left(x^{\rho}-\tau^{\rho}\right)^{\alpha-n+1}} d \tau
$$

if the integral exists.

Definition 2.2 ([19]). Let $n=\lceil\operatorname{Re}(\alpha)\rceil$. If $\mathrm{f} \in A C_{\mu}^{n}[a, b]$, then Caputo type generalized fractional derivative, ${ }_{c}^{\rho} D_{a^{+}}^{\alpha}$ is defined as

$$
{ }_{c}^{\rho} D_{a^{+}}^{\alpha} \mathrm{f}(x)=\left({ }^{\rho} D_{a^{+}}^{\alpha}\left[\mathrm{f}(t)-\sum_{k=0}^{n-1} \frac{\mu^{k} f(a)}{k !}\left(\frac{t^{\rho}-a^{\rho}}{\rho}\right)^{k}\right]\right)(x),
$$

where $\mu=x^{1-\rho} \frac{d}{d x}$.

Definition 2.3 ([30]). The map $\theta$ is said to be a nonnegative continuous concave functional on a cone $P$ of a real Banach space $E$ provided that $\theta: P \rightarrow[0, \infty)$ is continuous and $\theta(t x+(1-t) y) \geq t \theta(x)+(1-t) \theta(y)$ for all $x, y \in P$ and $t \in[0,1]$.

Lemma 2.4 ([30]). Let $P$ be a cone in a real Banach space $E, P_{c}=\{x \in P:\|x\| \leq c\}, \theta$ a nonnegative continuous functional on $P$ such that $\theta(x) \leq\|x\|$ for all $x \in P_{c}$, and $P(\theta, b, d)=\{x \in P: b \leq \theta(x),\|x\| \leq d\}$. Suppose $F: \bar{P}_{c} \rightarrow \bar{P}_{c}$ is completely continuous and there exist constants $0<a<b<d \leq c$ such that

$\left(C_{1}\right)\{x \in P(\theta, b, d): \theta(x)>b\} \neq \phi$ and $\theta(F x)$ for $x \in P(\theta, b, d)$;

$\left(C_{2}\right)\|F x\|<a$ for $x \leq a$;

$\left(C_{3}\right) \theta(F x)>b$ for $x \in P(\theta, b, c)$ with $\|F x\|>d$.

Then $F$ has at least three fixed points $x_{1}, x_{2}, x_{3}$ with $\left\|x_{1}\right\|<a, b<\theta\left(x_{2}\right), a<\left\|x_{3}\right\|$ with $\theta\left(x_{3}\right)=b$. 
Lemma 2.5 ([14]). Let $E$ be a Banach space and $P \subseteq E$ be a cone. Assume $\Omega_{1}$ and $\Omega_{2}$ are open discs contained in $E$ with $0 \in \Omega_{1}$, and $\overline{\Omega_{1}} \subset \Omega_{2}$. Furthermore, assume that $T: P \cap\left(\bar{\Omega}_{2} / \Omega_{1}\right) \rightarrow P$ be completely continuous operator such that either

(i) $\|T u\| \leq\|u\|$ for $u \in K \cap \partial \Omega_{1}$ and $\|T u\| \geq\|u\|$ for $u \in K \cap \partial \Omega_{2}$, or

(ii) $\|T u\| \geq\|u\|$ for $u \in K \cap \partial \Omega_{1}$ and $\|T u\| \leq\|u\|$ for $u \in K \cap \partial \Omega_{2}$.

Then $T$ has at least one fixed point in $K \cap\left(\bar{\Omega}_{2} / \Omega_{1}\right)$.

\section{Caputo type generalized fractional differential equations}

In this section, we consider the following two Caputo type boundary value problems of generalized fractional derivatives:

$$
\begin{gathered}
{ }_{c}^{\rho} D_{0^{+}}^{\beta}\left(\phi_{p}\left({ }_{c}^{\rho} D_{0^{+}}^{\alpha} u(t)\right)\right)+f(t, u(t))=0,1<\rho \leq 2,2<\alpha<3,0<\beta \leq 1, t \in(0,1), \\
u(0)=u(1)=u^{\prime \prime}(0)=0, \quad{ }_{c}^{\rho} D_{0^{+}}^{\alpha} u(0)=0,
\end{gathered}
$$

and

$$
\begin{aligned}
{ }_{c}^{\rho} D_{0^{+}}^{\beta}\left(\phi_{p}\left({ }_{c}^{\rho} D_{0^{+}}^{\alpha} u(t)\right)\right)-f(t, u(t)) & =0,0<\rho \leq 1,1<\alpha \leq 2,0<\beta \leq 1, t \in(0,1), \\
u(0)+u^{\prime}(0)=0, \quad u(1)+u^{\prime}(1) & =0, \quad{ }_{c}^{\rho} D_{0^{+}}^{\alpha} u(0)=0,
\end{aligned}
$$

where ${ }_{c}^{\rho} D_{0^{+}}^{\alpha},{ }_{c}^{\rho} D_{0^{+}}^{\beta}$ denote the generalized fractional derivatives, $\phi_{p}(s)=|s|^{p-2} s, p>$ $1, \phi_{p}^{-1}=\phi_{q}, \frac{1}{p}+\frac{1}{q}=1$, and $f:[0,1] \times[0,+\infty) \rightarrow[0,+\infty)$ is continuous.

Lemma 3.1. Let $y \in C[0,1]$ and $2<\alpha<3$. Then the boundary value problem

$$
\begin{aligned}
{ }_{c}^{\rho} D_{0^{+}}^{\alpha} u(t)+y(t) & =0,1<\rho \leq 2, t \in(0,1), \\
u(0)=u(1) & =u^{\prime \prime}(0)=0,
\end{aligned}
$$

where ${ }_{c}^{\rho} D_{a^{+}}^{\alpha}$ denotes the generalized fractional derivative, has a unique solution

$$
u(t)=\int_{0}^{1} G(t, s) y(s) d s,
$$

where

$$
G(t, s)=\frac{\rho^{1-\alpha} s^{\rho-1}}{\Gamma(\alpha)} \begin{cases}t^{2 \rho}\left(1-s^{\rho}\right)^{\alpha-1}-\left(t^{\rho}-s^{\rho}\right)^{\alpha-1}, & 0 \leq s \leq t \leq 1, \\ t^{2 \rho}\left(1-s^{\rho}\right)^{\alpha-1}, & 0 \leq t \leq s \leq 1 .\end{cases}
$$

Lemma 3.2. The boundary value problem for the generalized fractional differential equations (3.1) has a unique solution

$$
u(t)=\int_{0}^{1} G(t, s) \phi_{q}\left(\frac{\rho^{1-\beta}}{\Gamma(\beta)} \int_{0}^{s}\left(s^{\rho}-\tau^{\rho}\right)^{\beta-1} \tau^{\rho-1} f(\tau, u(\tau) d \tau) d s\right),
$$

where $G(t, s)$ is defined as (3.4).

Proof. From Lemma 3.1 and the boundary value problem (3.1), we have

$$
\begin{aligned}
{ }^{\rho} I_{0^{+} c}^{\beta} D_{0^{+}}^{\beta}\left(\phi\left({ }_{c}^{\rho} D_{0^{+}}^{\alpha} u(t)\right)\right. & =-{ }^{\rho} I_{0^{+}}^{\beta} f(t, u(t)) \\
& =\phi_{p}\left({ }_{c}^{\rho} D_{0^{+}}^{\alpha} u(t)-\phi_{p}\left({ }_{c}^{\rho} D_{0^{+}}^{\alpha} u(0)\right)\right. \\
& =\phi_{p}\left({ }_{c}^{\rho} D_{0^{+}}^{\alpha} u(t)\right) .
\end{aligned}
$$

This implies

$$
\phi_{p}\left({ }_{c}^{\rho} D_{0^{+}}^{\alpha} u(t)\right)=-{ }^{\rho} I_{0^{+}}^{\beta} f(t, u(t)) .
$$

So we have

$$
{ }_{c}^{\rho} D_{0^{+}}^{\alpha} u(t)=-\phi_{q}\left({ }^{\rho} I_{0^{+}}^{\beta} f(t, u(t))\right) .
$$


Thus (3.1) is equivalent to the following problem:

$$
\begin{array}{r}
{ }_{c}^{\rho} D_{0^{+}}^{\alpha} u(t)+\phi_{q}\left({ }^{\rho} I_{0^{+}}^{\beta} f(t, u(t))\right)=0, t \in(0,1), \\
\left.u(0)=u(1)=u^{\prime \prime}(0)=0,{ }_{c}^{\rho} D_{0^{+}}^{\alpha} u(0)\right)=0 .
\end{array}
$$

And so, Lemma 3.1 implies that (3.5) has a unique solution

$$
u(t)=\int_{0}^{1} G(t, s) \phi_{q}\left(\frac{\rho^{1-\beta}}{\Gamma(\beta)} \int_{0}^{s}\left(s^{\rho}-\tau^{\rho}\right)^{\beta-1} \tau^{\rho-1} f(\tau, u(\tau) d \tau) d s\right),
$$

where $G(t, s)$ is defined as (3.4).

Lemma 3.3. The Green's function defined by (3.4) is continuous on $[0,1] \times[0,1]$. Then $G(t, s)$ also has the following properties:

(i) $G(t, s)>0$ for $t, s \in(0,1)$.

(ii) $\left(t^{2 \rho}-t^{\rho(\alpha-1)}\right)\left(1-s^{\rho}\right)^{\alpha-1} \leq \frac{\Gamma(\alpha)}{\rho^{1-\alpha}} G(t, s) \leq\left(1-s^{\rho}\right)^{\alpha-1}$ for $t, s \in(0,1)$.

Proof. Since $2<\alpha<3$, then

(i) $t^{2 \rho}\left(1-s^{\rho}\right)^{\alpha-1}-\left(t^{\rho}-s^{\rho}\right)^{\alpha-1} \geq t^{2 \rho}\left(1-s^{\rho}\right)^{\alpha-1}-\left(t^{\rho}-s^{\rho} t^{\rho}\right)^{\alpha-1}$

$$
=\left(t^{2 \rho}-t^{\rho(\alpha-1)}\right)\left(1-s^{\rho}\right)^{\alpha-1}>0, t, s \in(0,1) .
$$

So, we have $G(t, s)>0$ for $t \in(0,1)$.

(ii) $\quad\left(1-s^{\rho}\right)^{\alpha-1} t^{2 \rho} \leq\left(1-s^{\rho}\right)^{\alpha-1} \Longrightarrow\left(1-s^{\rho}\right)^{\alpha-1} t^{2 \rho}-\left(t^{\rho}-s^{\rho}\right)^{\alpha-1} \leq\left(1-s^{\rho}\right)^{\alpha-1}$

$$
\Longrightarrow \frac{\Gamma(\alpha)}{\rho^{1-\alpha}} G(t, s) \leq\left(1-s^{\rho}\right)^{\alpha-1} \text {. }
$$

From $(i)$ and $(i i)$, we have

$$
\left(t^{2 \rho}-t^{\rho(\alpha-1)}\right)\left(1-s^{\rho}\right)^{\alpha-1} \leq \frac{\Gamma(\alpha)}{\rho^{1-\alpha}} G(t, s) \leq\left(1-s^{\rho}\right)^{\alpha-1} .
$$

Lemma 3.4. Let $y(t) \in C[0,1]$ and $1<\alpha \leq 2$. Then the boundary value problem

$$
\begin{gathered}
{ }_{c}^{\rho} D_{0^{+}}^{\alpha} u(t)-y(t)=0, \quad 0<\rho \leq 1, t \in(0,1), \\
u(0)+u^{\prime}(0)=0, \quad u(1)+u^{\prime}(1)=0,
\end{gathered}
$$

where ${ }_{c}^{\rho} D_{a^{+}}^{\alpha}$ denotes the generalized fractional derivative, has a unique solution

$$
u(t)=\int_{0}^{1} G(t, s) y(s) d s,
$$

where

$$
G(t, s)=\frac{\rho^{1-\alpha} s^{\rho-1}}{\Gamma(\alpha-1)} \begin{cases}\left(1-t^{\rho}\right)\left[\left(1-s^{\rho}\right)^{\alpha-2}+\frac{1}{\rho(\alpha-1)}\left(1-s^{\rho}\right)^{\alpha-1}\right] & \\ +\frac{1}{\alpha-1}\left(t^{\rho}-s^{\rho}\right)^{\alpha-1}, & 0 \leq s \leq t \leq 1, \\ \left(1-t^{\rho}\right)\left[\left(1-s^{\rho}\right)^{\alpha-2}+\frac{1}{\rho(\alpha-1)}\left(1-s^{\rho}\right)^{\alpha-1}\right], & 0 \leq t \leq s \leq 1 .\end{cases}
$$

Lemma 3.5. The boundary value problem for the generalized fractional differential equations (3.2) has a unique solution

$$
u(t)=\int_{0}^{1} G(t, s) \phi_{q}\left(\frac{\rho^{1-\beta}}{\Gamma(\beta)} \int_{0}^{s}\left(s^{\rho}-\tau^{\rho}\right)^{\beta-1} \tau^{\rho-1} f(\tau, u(\tau) d \tau) d s\right),
$$

where $G(t, s)$ is defined as (3.8).

Lemma 3.6. Let $y(t) \in C[0,1]$ be a given function, then function $G(t, s)$ defined by (3.8) has the following properties:

$A_{1} . G(t, s) \in C([0,1] \times[0,1])$, and $G(t, s)>0$ for $t, s \in(0,1)$; 
$A_{2}$. There exists a positive function $\gamma \in C(0,1)$ such that

$$
\begin{aligned}
\min _{1 / 4 \leq t \leq 3 / 4} G(t, s) & \geq \gamma(s) M(s), \quad s \in(0,1), \\
\max _{0 \leq t \leq 1} G(t, s) & \leq M(s),
\end{aligned}
$$

where

$$
M(s):=\frac{\rho^{1-\alpha} s^{\rho-1}}{\Gamma(\alpha-1)}\left[\left(1-s^{\rho}\right)^{\alpha-2}+\frac{2}{\rho(\alpha-1)}\left(1-s^{\rho}\right)^{\alpha-1}\right], \quad s \in(0,1) .
$$

Proof. $\left(A_{1}\right)$ is obvious. We will prove $\left(A_{2}\right)$ only.

Let

$$
\begin{aligned}
& G_{1}(t, s)=\frac{\rho^{1-\alpha} s^{\rho-1}}{\Gamma(\alpha-1)}\left[\left(1-t^{\rho}\right)\left(\left(1-s^{\rho}\right)^{\alpha-2}+\frac{1}{\rho(\alpha-1)}\left(1-s^{\rho}\right)^{\alpha-1}\right)+\frac{1}{\alpha-1}\left(t^{\rho}-s^{\rho}\right)^{\alpha-1}\right], s \leq t, \\
& G_{2}(t, s)=\frac{\rho^{1-\alpha} s^{\rho-1}}{\Gamma(\alpha-1)}\left(1-t^{\rho}\right)\left[\left(1-s^{\rho}\right)^{\alpha-2}+\frac{1}{\rho(\alpha-1)}\left(1-s^{\rho}\right)^{\alpha-1}\right], t \leq s .
\end{aligned}
$$

For given $s \in(0,1), G_{2}(t, s)$ is decreasing with respect to $t$ and $G_{1}(t, s)$ is a continuous function for $1 / 4 \leq t \leq 3 / 4$. Thus, we have

$$
\begin{gathered}
G_{1}(t, s) \geq \frac{\rho^{1-\alpha} s^{\rho-1}}{\Gamma(\alpha-1)}\left(1-\left(\frac{3}{4}\right)^{\rho}\right)\left[\left(1-s^{\rho}\right)^{\alpha-2}+\frac{1}{\rho(\alpha-1)}\left(1-s^{\rho}\right)^{\alpha-1}\right], \text { for } 1 / 4 \leq t \leq 3 / 4, \\
\max _{0 \leq t \leq 1} G_{1}(t, s) \leq \frac{\rho^{1-\alpha} s^{\rho-1}}{\Gamma(\alpha-1)}\left[\left(1-s^{\rho}\right)^{\alpha-2}+\frac{2}{\alpha-1}\left(1-s^{\rho}\right)^{\alpha-1}\right], \\
\min _{1 / 4 \leq t \leq 3 / 4} G_{2}(t, s)=G_{2}(3 / 4, s)=\frac{\rho^{1-\alpha} s^{\rho-1}}{\Gamma(\alpha-1)}\left(1-\left(\frac{3}{4}\right)^{\rho}\right)\left[\left(1-s^{\rho}\right)^{\alpha-2}+\frac{1}{\rho(\alpha-1)}\left(1-s^{\rho}\right)^{\alpha-1}\right], \\
\max _{0 \leq t \leq 1} G_{2}(t, s)=G_{2}(0, s)=\frac{\rho^{1-\alpha} s^{\rho-1}}{\Gamma(\alpha-1)}\left[\left(1-s^{\rho}\right)^{\alpha-2}+\frac{1}{\rho(\alpha-1)}\left(1-s^{\rho}\right)^{\alpha-1}\right] \\
<\frac{\rho^{1-\alpha} s^{\rho-1}}{\Gamma(\alpha-1)}\left[\left(1-s^{\rho}\right)^{\alpha-2}+\frac{2}{\rho(\alpha-1)}\left(1-s^{\rho}\right)^{\alpha-1}\right] .
\end{gathered}
$$

Thus, we have

$$
\begin{gathered}
\min _{1 / 4 \leq t \leq 3 / 4} G(t, s) \geq m(s)=\frac{\rho^{1-\alpha} s^{\rho-1}}{\Gamma(\alpha-1)}\left(1-\left(\frac{3}{4}\right)^{\rho}\right)\left[\left(1-s^{\rho}\right)^{\alpha-2}+\frac{1}{\rho(\alpha-1)}\left(1-s^{\rho}\right)^{\alpha-1}\right], \quad s \in(0,1), \\
\max _{0 \leq t \leq 1} G(t, s) \leq M(s)=\frac{\rho^{1-\alpha} s^{\rho-1}}{\Gamma(\alpha-1)}\left[\left(1-s^{\rho}\right)^{\alpha-2}+\frac{2}{\rho(\alpha-1)}\left(1-s^{\rho}\right)^{\alpha-1}\right], \quad s \in(0,1) .
\end{gathered}
$$

Let

$$
\gamma(s)=m(s) / M(s)=\left(1-\left(\frac{3}{4}\right)^{\rho}\right) \frac{\rho(\alpha-1)\left(1-s^{\rho}\right)^{\alpha-2}+\left(1-s^{\rho}\right)^{\alpha-1}}{\rho(\alpha-1)\left(1-s^{\rho}\right)^{\alpha-2}+2\left(1-s^{\rho}\right)^{\alpha-1}}, \quad s \in(0,1) .
$$

Remark 3.7. For the problems (3.1) and (3.2), when $\rho=1$, Green's functions and their properties are same as in [12], [31].

\section{Riemann-Liouville type generalized fractional differential equations}

In this section, we consider the following two Riemann-Liouville type boundary value problems of generalized fractional derivatives:

$$
\begin{gathered}
{ }^{\rho} D_{0^{+}}^{\beta}\left(\phi_{p}\left({ }^{\rho} D_{0^{+}}^{\alpha} u(t)\right)\right)+f(t, u(t))=0,1<\alpha \leq 2,0<\beta \leq 1, t \in(0,1), \\
u(0)=u(1)=0,{ }^{\rho} D_{0^{+}}^{\alpha} u(0)=0,
\end{gathered}
$$


and

$$
\begin{aligned}
{ }^{\rho} D_{0^{+}}^{\beta}\left(\phi_{p}\left({ }^{\rho} D_{0^{+}}^{\alpha} u(t)\right)\right)+f(t, u(t)) & =0,1<\alpha \leq 2,0<\beta \leq 1, t \in(0,1), \\
u(0) & =0, \beta u(\eta)=u(1), \quad{ }^{\rho} D_{0^{+}}^{\alpha} u(0)=0,
\end{aligned}
$$

where ${ }^{\rho} D_{0^{+}}^{\alpha},{ }^{\rho} D_{0^{+}}^{\beta}$ denote the generalized fractional derivatives, $\phi_{p}(s)=|s|^{p-2} s, p>$ $1, \phi_{p}^{-1}=\phi_{q}, \frac{1}{p}+\frac{1}{q}=1$, and $f:[0,1] \times[0,+\infty) \rightarrow[0,+\infty)$ is continuous.

Lemma 4.1. Let $y \in C[0,1]$ and $1<\alpha \leq 2$. Then the boundary value problem

$$
\begin{aligned}
{ }^{\rho} D_{0^{+}}^{\alpha} u(t)+y(t) & =0, t \in(0,1), \\
u(0)=u(1) & =0,
\end{aligned}
$$

where ${ }^{\rho} D_{a^{+}}^{\alpha}$ denotes the generalized fractional derivative, has a unique solution

$$
u(t)=\int_{0}^{1} G(t, s) y(s) d s,
$$

where

$$
G(t, s)=\frac{\rho^{1-\alpha} s^{\rho-1}}{\Gamma(\alpha)} \begin{cases}\left(t^{\rho}\left(1-s^{\rho}\right)\right)^{\alpha-1}-\left(t^{\rho}-s^{\rho}\right)^{\alpha-1}, & 0 \leq s \leq t \leq 1 \\ \left(t^{\rho}\left(1-s^{\rho}\right)\right)^{\alpha-1}, & 0 \leq t \leq s \leq 1 .\end{cases}
$$

Lemma 4.2. The boundary value problem (4.1) for the generalized fractional differential equations has unique solution

$$
u(t)=\int_{0}^{1} G(t, s) \phi_{q}\left(\frac{\rho^{1-\beta}}{\Gamma(\beta)} \int_{0}^{s}\left(s^{\rho}-\tau^{\rho}\right)^{\beta-1} \tau^{\rho-1} f(\tau, u(\tau) d \tau) d s\right),
$$

where $G(t, s)$ is defined as (4.4).

Lemma 4.3. The Green's function defined by (4.4) is continuous on $[0,1] \times[0,1]$. Then $G(t, s)$ has the following properties:

(i) $G(t, s)>0$ for $t, s \in(0,1)$.

(ii) $\frac{\Gamma(\alpha)}{\rho^{1-\alpha}} G(t, s) \leq\left(1-s^{\rho}\right)^{\alpha-1} s^{\rho-1}$ for $t, s \in(0,1)$.

(iii) $\min _{1 / 4 \leq t \leq 3 / 4} G(t, s) \geq k(s)\left(1-s^{\rho}\right)^{\alpha-1} s^{\rho-1}$ for $s \in(0,1)$.

Proof. (i) Since $t^{\rho}<1$ implies $\frac{s^{\rho}}{t^{\rho}}>s^{\rho}$ thus we obtain $1-\frac{s^{\rho}}{t^{\rho}}<1-s^{\rho}$ which gives $\left[t^{\rho}\left(1-s^{\rho}\right)\right]^{\alpha-1}-\left(t^{\rho}-s^{\rho}\right)^{\alpha-1}>0$ for $t \geq s$. And clearly, for $t \leq s$, we have $\left[t^{\rho}\left(1-s^{\rho}\right)\right]^{\alpha-1}>0$. Hence $G(t, s)>0$ for $t, s \in(0,1)$.

(ii) For $t \geq s, \quad t^{\rho(\alpha-1)} \leq 1$ yields $\left[t^{\rho}\left(1-s^{\rho}\right)\right]^{\alpha-1} \leq\left(1-s^{\rho}\right)^{\alpha-1}$ which implies $\left[t^{\rho}(1-\right.$ $\left.\left.s^{\rho}\right)\right]^{\alpha-1}-\left(t^{\rho}-s^{\rho}\right)^{\alpha-1} \leq\left(1-s^{\rho}\right)^{\alpha-1}$. Thus we obtain $\frac{\Gamma(\alpha)}{\rho^{1-\alpha}} G(t, s) \leq\left(1-s^{\rho}\right)^{\alpha-1} s^{\rho-1}$ for $t \geq s$.

And for $t \leq s$, again we obtain $\frac{\Gamma(\alpha)}{\rho^{1-\alpha}} G(t, s) \leq\left(1-s^{\rho}\right)^{\alpha-1} s^{\rho-1}$. Hence $\frac{\Gamma(\alpha)}{\rho^{1-\alpha}} G(t, s) \leq$ $\left(1-s^{\rho}\right)^{\alpha-1} s^{\rho-1}$ for $t, s \in(0,1)$.

(iii) Next, we observe that $G(t, s)$ is decreasing with respect to $t$ for $t \geq s$ and increasing with respect to $t$ for $t \leq s$. Now, we set

$$
\begin{aligned}
& G_{1}(t, s)=\frac{\rho^{1-\alpha}}{\Gamma(\alpha)} s^{\rho-1}\left[t^{\rho}\left(1-s^{\rho}\right)\right]^{\alpha-1}-\left(t^{\rho}-s^{\rho}\right)^{\alpha-1}, \quad 0 \leq s \leq t \leq 1, \\
& G_{2}(t, s)=\frac{\rho^{1-\alpha}}{\Gamma(\alpha)} s^{\rho-1}\left[t^{\rho}\left(1-s^{\rho}\right)\right]^{\alpha-1}, \quad 0 \leq t \leq s \leq 1 .
\end{aligned}
$$

First we show that $G_{1}(t, s)$ is decreasing with respect to $t$ for $t \geq s$.

Taking derivative of $G_{1}$ with respect to $t$, we have

$$
\begin{aligned}
& \frac{\partial G_{1}(t, s)}{\partial t}=\rho(\alpha-1)\left[t^{\rho(\alpha-1)-1}\left(1-s^{\rho}\right)^{\alpha-1}-t^{\rho-1}\left(t^{\rho}-s^{\rho}\right)^{\alpha-2}\right] \leq t^{\rho-1}\left[\left(1-s^{\rho}\right)^{\alpha-1}-\right. \\
& \left.\left(t^{\rho}-s^{\rho}\right)^{\alpha-2}\right] .
\end{aligned}
$$

Since $-1<\alpha-2 \leq 0,0<s^{\rho}<1$, we obtain $-s^{\rho}<0 \Longrightarrow t^{\rho}-s^{\rho}<t^{\rho}<1$. Also 
we have $t^{\rho}-s^{\rho} \geq 0$. Hence $0 \leq t^{\rho}-s^{\rho}<1$ which implies $\left(t^{\rho}-s^{\rho}\right)^{\alpha-2}>1$, and since $\left(1-s^{\rho}\right)<1$, we conclude that $\left[\left(1-s^{\rho}\right)^{\alpha-1}-\left(t^{\rho}-s^{\rho}\right)^{\alpha-2}\right]<0$.

We have

$$
\begin{aligned}
\min _{1 / 4 \leq t \leq 3 / 4} G(t, s) & =\left\{\begin{array}{l}
G_{1}(3 / 4, s), s \in(0,1 / 4], \\
\min \left\{G_{1}(3 / 4, s), G_{2}(1 / 4, s)\right\}, s \in[1 / 4,3 / 4], \\
G_{2}(1 / 4, s), s \in[3 / 4,1),
\end{array}\right. \\
& =\left\{\begin{array}{l}
G_{1}(3 / 4, s), s \in(0, k], \\
G_{2}(1 / 4, s), s \in[k, 1),
\end{array}\right.
\end{aligned}
$$

where $1 / 4<k<3 / 4$ is the unique solution of the equation $G_{1}(3 / 4, s)=G_{2}(1 / 4, s)$.

$$
k(s)=\left\{\begin{array}{l}
\frac{\left[(3 / 4)^{\rho}\left(1-s^{\rho}\right)\right]^{\alpha-1}-\left((3 / 4)^{\rho}-s^{\rho}\right)^{\alpha-1}}{\left(1-s^{\rho}\right)^{\alpha-1}}, s \in(0, k], \\
\left(\frac{1}{4}\right)^{\rho(\alpha-1)}, s \in[k, 1) .
\end{array}\right.
$$

Before stating the next lemma, let us introduce the following notation:

$$
\Lambda:=\frac{\rho^{1-\alpha} s^{\rho-1}}{\Gamma(\alpha)\left(1-\beta \eta^{\rho(\alpha-1)}\right)} .
$$

Lemma 4.4. Let $y(t) \in C[0,1]$ and $1<\alpha \leq 2$. Then the boundary value problem

$$
\begin{aligned}
& { }^{\rho} D_{0^{+}}^{\alpha} u(t)+y(t)=0, t \in(0,1), \\
& u(0)=0, \quad \beta u(\eta)=u(1),
\end{aligned}
$$

where $0<\beta \eta^{\alpha-1}<1,0<\eta<1$, and ${ }^{\rho} D_{0^{+}}^{\alpha}$ is the generalized fractional derivative, has a unique solution

$$
u(t)=\int_{0}^{1} G(t, s) y(s) d s
$$

where

$$
G(t, s)=\Lambda\left\{\begin{array}{l}
{\left[t^{\rho}\left(1-s^{\rho}\right)\right]^{\alpha-1}-\left(1-\beta \eta^{\rho(\alpha-1)}\right)\left(t^{\rho}-s^{\rho}\right)^{\alpha-1}, 0<\eta \leq s \leq t \leq 1,} \\
{\left[t^{\rho}\left(1-s^{\rho}\right)\right]^{\alpha-1}-\beta t^{\rho(\alpha-1)}\left(\eta^{\rho}-s^{\rho}\right)^{\alpha-1}, 0 \leq t \leq s \leq \eta<1,} \\
{\left[t^{\rho}\left(1-s^{\rho}\right)\right]^{\alpha-1}, 0 \leq t \leq s \leq 1, \eta \leq s,} \\
{\left[t^{\rho}\left(1-s^{\rho}\right)\right]^{\alpha-1}-\beta t^{\rho(\alpha-1)}\left(\eta^{\rho}-s^{\rho}\right)^{\alpha-1}-\left(1-\beta \eta^{\rho(\alpha-1)}\right)\left(t^{\rho}-s^{\rho}\right)^{\alpha-1}} \\
\quad 0 \leq s \leq t \leq 1, s \leq \eta .
\end{array}\right.
$$

Lemma 4.5. The boundary value problem for the generalized fractional differential equations (4.2) has a unique solution

$$
u(t)=\int_{0}^{1} G(t, s) \phi_{q}\left(\frac{\rho^{1-\beta}}{\Gamma(\beta)} \int_{0}^{s}\left(s^{\rho}-\tau^{\rho}\right)^{\beta-1} \tau^{\rho-1} f(\tau, u(\tau) d \tau) d s\right),
$$

where $G(t, s)$ is defined as (4.7).

Lemma 4.6. The function $G(t, s)$ defined by (4.7) satisfies $G(t, s)>0$ for $t, s \in(0,1)$.

Proof. For $0<s \leq t \leq 1$, let

$$
\begin{aligned}
g_{1}(t, s) & =\left[t^{\rho}\left(1-s^{\rho}\right)\right]^{\alpha-1}-\beta t^{\rho(\alpha-1)}\left(\eta^{\rho}-s^{\rho}\right)^{\alpha-1}-\left(1-\beta \eta^{\rho(\alpha-1)}\right)\left(t^{\rho}-s^{\rho}\right)^{\alpha-1} \\
& =t^{\rho(\alpha-1)}\left[\left(1-s^{\rho}\right)^{\alpha-1}-\beta\left(\eta^{\rho}-s^{\rho}\right)^{\alpha-1}-\left(1-\beta \eta^{\rho(\alpha-1)}\right)\left(1-\left(\frac{s}{t}\right)^{\rho}\right)^{\alpha-1}\right]
\end{aligned}
$$


Setting $g(t)=\left(1-s^{\rho}\right)^{\alpha-1}-\beta\left(\eta^{\rho}-s^{\rho}\right)^{\alpha-1}-\left(1-\beta \eta^{\rho(\alpha-1)}\right)\left(1-\left(\frac{s}{t}\right)^{\rho}\right)^{\alpha-1}$, we obtain

$$
g^{\prime}(t)=-\rho(\alpha-1)\left(1-\beta \eta^{\rho(\alpha-1)}\right)\left(1-\left(\frac{s}{t}\right)^{\rho}\right)^{\alpha-2} \frac{s^{\rho}}{t^{\rho+1}} \leq 0 .
$$

Thus $g(t)$ is decreasing on $(0,1)$. And

$$
\begin{aligned}
g(1) & =\left(1-s^{\rho}\right)^{\alpha-1}-\beta\left(\eta^{\rho}-s^{\rho}\right)^{\alpha-1}-\left(1-\beta \eta^{\rho(\alpha-1)}\right)\left(1-s^{\rho}\right)^{\alpha-1} \\
& =-\beta\left(\eta^{\rho}-s^{\rho}\right)^{\alpha-1}+\beta \eta^{\rho(\alpha-1)}\left(1-s^{\rho}\right)^{\alpha-1} \\
& =\beta \eta^{\rho(\alpha-1)}\left[\left(1-s^{\rho}\right)^{\alpha-1}-\left(1-\left(\frac{s}{\eta}\right)^{\rho}\right)^{\alpha-1}\right]>0 .
\end{aligned}
$$

Therefore, $g_{1}(t, s)>0$ for $0<s<t \leq 1, s \leq \eta$. As also for $t=s, g_{1}(t, s)>0$. So, $g_{1}(t, s)>0$ for $t, s \in(0,1)$.

Now let

$$
\begin{aligned}
& g_{2}(t, s)=\left[t^{\rho}\left(1-s^{\rho}\right)\right]^{\alpha-1}-\left(1-\beta \eta^{\rho(\alpha-1)}\right)\left(t^{\rho}-s^{\rho}\right)^{\alpha-1}, 0<\eta \leq s \leq t \leq 1, \\
& g_{3}(t, s)=\left[t^{\rho}\left(1-s^{\rho}\right)\right]^{\alpha-1}-\beta t^{\rho(\alpha-1)}\left(\eta^{\rho}-s^{\rho}\right)^{\alpha-1}, 0 \leq t \leq s \leq \eta<1, \\
& g_{4}(t, s)=\left[t^{\rho}\left(1-s^{\rho}\right)\right]^{\alpha-1}, 0 \leq t \leq s \leq 1, \eta \leq s,
\end{aligned}
$$

then in the same way as above, it is easy to show that $g_{2}(t, s)>0, g_{3}(t, s)>0, g_{4}(t, s)>0$. Hence, for $t, s \in(0,1), G(t, s)>0$.

Remark 4.7. For the problems (4.1) and (4.2), when $\rho=1$, Green's functions and their properties are same as in [6], [7].

\section{Applications}

Let $E=C[0,1]$ be the Banach space equipped with the norm $\|u\|=\max _{t \in[0,1]}|u(t)|$. We define $P \subset E$ as

$$
P=\left\{u \in E: u(t) \geq \gamma\|u\|, t \in\left[\frac{1}{4}, \frac{3}{4}\right]\right\},
$$

where $\gamma=\min _{t \in\left[\frac{1}{4}, \frac{3}{4}\right]}\left(t-t^{\alpha-1}\right)$.

Let $\theta$ be the nonnegative continuous concave functional on the cone $P$ defined by

$$
\theta(u)=\min _{1 / 4 \leq t \leq 3 / 4}|u(t)|,
$$

then clearly $\theta(u) \leq\|u\|$ for each $u \in P$.

Let $T: E \rightarrow E$ be an operator defined as

$$
T u(t)=\int_{0}^{1} G(t, s) \phi_{q}\left(\frac{\rho^{1-\beta}}{\Gamma(\beta)} \int_{0}^{s}\left(s^{\rho}-\tau^{\rho}\right)^{\beta-1} \tau^{\rho-1} f(\tau, u(\tau) d \tau) d s\right),
$$

then $u \in E$ is a solution of (5.2) if and only if $u \in E$ is a solution of (3.1).

Lemma 5.1. Let $T$ be defined as in (5.2) and $P$ in (5.1). Then $T: P \rightarrow P$ is completely continuous.

Proof. Let $u \in$ P. From (3.6), we have

$$
\|T u\| \leq \frac{\rho^{1-\alpha}}{\Gamma(\alpha)} \int_{0}^{1}\left(1-s^{\rho}\right)^{\alpha-1} \phi_{q}\left(\frac{\rho^{1-\beta}}{\Gamma(\beta)} \int_{0}^{s}\left(s^{\rho}-\tau^{\rho}\right)^{\beta-1} \tau^{\rho-1} f(\tau, u(\tau)) d \tau\right) d s .
$$


From (5.3) and from (3.6), we obtain

$$
\begin{aligned}
T u(t) & \geq \frac{\rho^{1-\alpha}}{\Gamma(\alpha)} \int_{0}^{1}\left(t-t^{\alpha-1}\right)\left(1-s^{\rho}\right)^{\alpha-1} \phi_{q}\left(\frac{\rho^{1-\beta}}{\Gamma(\beta)} \int_{0}^{s}\left(s^{\rho}-\tau^{\rho}\right)^{\beta-1} \tau^{\rho-1} f(\tau, u(\tau) d \tau) d s\right. \\
& =\left(t-t^{\alpha-1}\right) \frac{\rho^{1-\alpha}}{\Gamma(\alpha)} \int_{0}^{1}\left(1-s^{\rho}\right)^{\alpha-1} \phi_{q}\left(\frac{\rho^{1-\beta}}{\Gamma(\beta)} \int_{0}^{s}\left(s^{\rho}-\tau^{\rho}\right)^{\beta-1} \tau^{\rho-1} f(\tau, u(\tau) d \tau) d s\right. \\
& \geq\left(t-t^{\alpha-1}\right)\|T u\| \geq \gamma\|T u\|, \quad t \in\left[\frac{1}{4}, \frac{3}{4}\right] .
\end{aligned}
$$

Since $f$ is continuous, it is easy to prove that $T$ is continuous. Now we only need to show that $T$ is compact.

Let $\Omega \subset P$ be bounded that there exists a constant $M>0$ such that $\|u\| \leq M$ for $u \in \Omega$. Let $L=\max _{t \in[0,1], u \in[0, M]}|f(t, u)|+1$, then for $u \in \Omega$, we have

$$
\begin{aligned}
|T u| & \leq \frac{\rho^{1-\alpha}}{\Gamma(\alpha)} \int_{0}^{1}\left(1-s^{\rho}\right)^{\alpha-1} \phi_{q}\left(\frac{\rho^{1-\beta}}{\Gamma(\beta)} \int_{0}^{s}\left(s^{\rho}-\tau^{\rho}\right)^{\beta-1} \tau^{\rho-1} f(\tau, u(\tau)) d \tau\right) d s \\
& \leq \frac{\rho^{1-\alpha}}{\Gamma(\alpha)} \int_{0}^{1}\left(1-s^{\rho}\right)^{\alpha-1} \phi_{q}\left(\frac{L \rho^{1-\beta}}{\Gamma(\beta)} \int_{0}^{s}\left(s^{\rho}-\tau^{\rho}\right)^{\beta-1} \tau^{\rho-1} d \tau\right) d s \\
& =\frac{\rho^{1-\alpha}}{\Gamma(\alpha)} \int_{0}^{1}\left(1-s^{\rho}\right)^{\alpha-1} \phi_{q}\left(\frac{L s^{\beta \rho}}{\rho^{\beta} \Gamma(\beta+1)}\right) d s \\
& =\frac{\rho^{1-\alpha}}{\Gamma(\alpha)} \phi_{q}\left(\frac{L}{\rho^{\beta} \Gamma(\beta+1)}\right) \int_{0}^{1}\left(1-s^{\rho}\right)^{\alpha-1} \phi_{q}\left(s^{\beta \rho}\right) d s .
\end{aligned}
$$

Hence $T(\Omega)$ is uniformly bounded. Further for any $u \in \Omega, t \in[0,1]$, and $0 \leq t_{1} \leq t_{2} \leq 1$, we have

$$
\left|T u\left(t_{1}\right)-T u\left(t_{2}\right)\right|=\int_{0}^{1}\left|G\left(t_{1}, s\right)-G\left(t_{2}, s\right)\right| \phi_{q}\left(\frac{\rho^{1-\beta}}{\Gamma(\beta)} \int_{0}^{s}\left(s^{\rho}-\tau^{\rho}\right)^{\beta-1} \tau^{\rho-1} f(\tau, u(\tau)) d \tau\right) d s,
$$

where

$$
\begin{aligned}
G\left(t_{1}, s\right)-G\left(t_{2}, s\right) & =\frac{\rho^{1-\alpha}}{\Gamma(\alpha)} s^{\rho-1} \\
& \left\{\begin{array}{l}
{\left[\left(t_{1}-t_{2}\right)\left(1-s^{\rho}\right)^{\alpha-1}-\left(\left(t_{1}^{\rho}-s^{\rho}\right)^{\alpha-1}-\left(t_{2}^{\rho}-s^{\rho}\right)^{\alpha-1}\right)\right], \quad 0 \leq s \leq t \leq 1,} \\
{\left[\left(t_{1}-t_{2}\right)\left(1-s^{\rho}\right)^{\alpha-1}\right], \quad 0 \leq t \leq s \leq 1 .}
\end{array}\right.
\end{aligned}
$$

Let $\xi \in\left(t_{1}, t_{2}\right)$, by mean value theorem, we have

$$
\left(t_{1}^{\rho}-s^{\rho}\right)^{\alpha-1}-\left(t_{2}^{\rho}-s^{\rho}\right)^{\alpha-1}=h^{\prime}(\xi)\left(t_{1}-t_{2}\right),
$$

therefore we obtain

$$
\begin{aligned}
G\left(t_{1}, s\right)-G\left(t_{2}, s\right) & =\frac{\rho^{1-\alpha}}{\Gamma(\alpha)} s^{\rho-1}\left(t_{1}-t_{2}\right)\left\{\begin{array}{l}
{\left[\left(1-s^{\rho}\right)^{\alpha-1}-h^{\prime}(\xi)\right], \quad 0 \leq s \leq t \leq 1} \\
{\left[\left(1-s^{\rho}\right)^{\alpha-1}\right], \quad 0 \leq t \leq s \leq 1}
\end{array}\right. \\
& =\left(t_{1}-t_{2}\right) G_{s}
\end{aligned}
$$

where $G_{s}=\frac{G\left(t_{1}, s\right)-G\left(t_{2}, s\right)}{\left(t_{1}-t_{2}\right)}$.

Thus, we get

$$
\left|T u\left(t_{1}\right)-T u\left(t_{2}\right)\right| \leq\left|t_{1}-t_{2}\right| \phi_{q}\left(\frac{L}{\rho^{\beta} \Gamma(\beta+1)}\right) \int_{0}^{1} G_{s} \phi_{q}\left(s^{\beta \rho}\right) d s .
$$

That implies $T(\Omega)$ is equicontinuous. By Arzela-Ascoli theorem, $T: P \rightarrow P$ is completely continuous. 
Theorem 5.2. Assume $f:[0,1] \times[0,+\infty] \rightarrow[0,+\infty]$ is continuous and there exist distinct $\rho_{1}, \rho_{2}>0$ such that

$$
\begin{aligned}
& \left(H_{1}\right) f(t, u) \leq \frac{\Gamma(\beta)}{\rho^{1-\beta}} \phi_{p}\left(\rho_{1} N\right) \text { for }(t, u) \in[0,1] \times\left[0, \rho_{1}\right], \\
& \left(H_{2}\right) f(t, u) \geq \frac{\Gamma(\beta)}{\rho^{1-\beta}} \phi_{p}\left(\rho_{2} M\right) \text { for }(t, u) \in[1 / 4,3 / 4] \times\left[0, \rho_{2}\right] .
\end{aligned}
$$

Then boundary value problem (3.1) has at least one positive solution u such that $\rho_{1} \leq$ $\|u\| \leq \rho_{2}$.

Proof. Let $0<\rho_{1}<\rho_{2}$. And for $u \in P$, by Lemma 5.1, Tu $\in P$ and $T$ is completely continuous operator. Now let $\Omega_{1}=\left\{u \in P:\|u\|<\rho_{1}\right\}$. For $u \in \partial \Omega_{1}$, we have, $\|u\|=\rho_{1}$. So, for $u \in P \cap \partial \Omega_{1}$, we get

$$
\begin{aligned}
\|T u\| & \leq \frac{\rho^{1-\alpha}}{\Gamma(\alpha)} \int_{0}^{1}\left(1-s^{\rho}\right)^{\alpha-1} \phi_{q}\left(\frac{\rho^{1-\beta}}{\Gamma(\beta)} \int_{0}^{s}\left(s^{\rho}-\tau^{\rho}\right)^{\beta-1} \tau^{\rho-1} f(\tau, u(\tau)) d \tau\right) d s \\
& \leq \frac{\rho^{1-\alpha}}{\Gamma(\alpha)} \rho_{1} N \int_{0}^{1}\left(1-s^{\rho}\right)^{\alpha-1} \phi_{q}\left(\int_{0}^{s}\left(s^{\rho}-\tau^{\rho}\right)^{\beta-1} \tau^{\rho-1} d \tau\right) d s \\
& =\frac{\rho^{1-\alpha}}{\Gamma(\alpha)} \rho_{1} N \int_{0}^{1}\left(1-s^{\rho}\right)^{\alpha-1} \phi_{q}\left(\frac{s^{\beta \rho}}{\beta \rho}\right) d s=\rho_{1}=\|u\|,
\end{aligned}
$$

where $N=\frac{\Gamma(\alpha)}{\rho^{1-\alpha}}\left(\int_{0}^{1}\left(1-s^{\rho}\right)^{\alpha-1} \phi_{q}\left(\frac{s^{\beta \rho}}{\beta \rho}\right) d s\right)^{-1}$.

On the other hand, put $\Omega_{2}=\left\{u \in P:\|u\|<\rho_{2}\right\}$. For $u \in \partial \Omega_{2},\|u\|=\rho_{2}$. So, for $u \in P \cap \partial \Omega_{2}$, we obtain, for $t \in[1 / 4,3 / 4]$,

$$
\begin{aligned}
T u(t) & =\int_{0}^{1} G(t, s) \phi_{q}\left(\frac{\rho^{1-\beta}}{\Gamma(\beta)} \int_{0}^{s}\left(s^{\rho}-\tau^{\rho}\right)^{\beta-1} \tau^{\rho-1} f(\tau, u(\tau)) d \tau\right) d s \\
& \geq \frac{\rho^{1-\alpha}}{\Gamma(\alpha)} \int_{1 / 4}^{3 / 4}\left(t-t^{\alpha-1}\right)\left(1-s^{\rho}\right)^{\alpha-1} \phi_{q}\left(\frac{\rho^{1-\beta}}{\Gamma(\beta)} \int_{0}^{s}\left(s^{\rho}-\tau^{\rho}\right)^{\beta-1} \tau^{\rho-1} f(\tau, u(\tau)) d \tau\right) d s \\
& \geq \frac{\rho^{1-\alpha}}{\Gamma(\alpha)} \rho_{2} M \int_{1 / 4}^{3 / 4} \gamma\left(1-s^{\rho}\right)^{\alpha-1} \phi_{q}\left(\frac{s^{\beta \rho}}{\beta \rho}\right) d s=\rho_{2}=\|u\|,
\end{aligned}
$$

where $M=\frac{\Gamma(\alpha)}{\gamma \rho^{1-\alpha}}\left(\int_{1 / 4}^{3 / 4}\left(1-s^{\rho}\right)^{\alpha-1} \phi_{q}\left(\frac{s^{\beta \rho}}{\beta \rho}\right) d s\right)^{-1}$.

So, we have $\|T u\| \geq\|u\|$, for $u \in P \cap \partial \Omega_{2}$. Hence by Lemma $2.5, T$ has at least one fixed point in $P \cap\left(\bar{\Omega}_{2} / \Omega_{1}\right)$.

Theorem 5.3. Suppose that $f(t, u)$ is a nonnegative continuous function on $[0,1] \times[0, \infty)$ and there exist constants $0<a<b<c$ such that the following assumptions hold:

$$
\begin{aligned}
& \left(A_{1}\right) f(t, u)<\frac{\Gamma(\beta)}{\rho^{1-\beta}} \phi_{p}(N a), \text { for }(t, u) \in[0,1] \times[0, a] ; \\
& \left(A_{2}\right) f(t, u) \geq \frac{\Gamma(\beta)}{\rho^{1-\beta}} \phi_{p}(M b), \text { for }(t, u) \in[1 / 4,3 / 4] \times[b, c] ; \\
& \left(A_{3}\right) f(t, u) \leq \frac{\Gamma(\beta)}{\rho^{1-\beta}} \phi_{p}(N c), \text { for }(t, u) \in[0,1] \times[0, c] .
\end{aligned}
$$

Then the BVP (3.1) has at least three positive solutions $u_{1}, u_{2}$, and $u_{3}$ with

$$
\begin{gathered}
\max _{t \in[0,1]}\left|u_{1}(t)\right|<a, \quad b<\min _{t \in[1 / 4,3 / 4]}\left|u_{2}(t)\right|<\max _{t \in[0,1]}\left|u_{2}(t)\right| \leq c, \\
a<\max _{t \in[0,1]}\left|u_{3}(t)\right| \leq c, \min _{t \in[1 / 4,3 / 4]}\left|u_{3}(t)\right|<b .
\end{gathered}
$$


Proof. If $u \in \bar{P}_{c}$, then $\|u\| \leq c$. Assumption $\left(A_{3}\right)$ implies $f(t, u) \leq \frac{\Gamma(\beta)}{\rho^{1-\beta}} \phi_{p}(N c)$ for $0 \leq t \leq 1$. Consequently,

$$
\begin{aligned}
\|T u\| & =\max _{0 \leq t \leq 1}\left|\int_{0}^{1} G(t, s) \phi_{q}\left(\frac{\rho^{1-\beta}}{\Gamma(\beta)} \int_{0}^{s}\left(s^{\rho}-\tau^{\rho}\right)^{\beta-1} \tau^{\rho-1} f(\tau, u(\tau)) d \tau\right) d s\right| \\
& \leq N c \frac{\rho^{1-\alpha}}{\Gamma(\alpha)} \int_{0}^{1}\left(1-s^{\rho}\right)^{\alpha-1} \phi_{q}\left(\frac{s^{\beta \rho}}{\beta \rho}\right) d s \\
& \leq N c \phi_{q}\left(\frac{1}{\beta \rho}\right) \frac{\rho^{1-\alpha}}{\Gamma(\alpha)} \int_{0}^{1}\left(1-s^{\rho}\right)^{\alpha-1} \phi_{q}\left(s^{\beta \rho}\right) d s=c .
\end{aligned}
$$

This implies $T: \bar{P}_{c} \rightarrow \bar{P}_{c}$. Using the same argument as in the proof of the Lemma 5.1, we can show that $T: \bar{P}_{c} \rightarrow \bar{P}_{c}$ is completely continuous. In the same way, if $u \in \bar{P}_{a}$, then assumption $\left(A_{1}\right)$ yields $f(t, u)<\frac{\Gamma(\beta)}{\rho^{1-\beta}} \phi_{p}(N a), 0 \leq t \leq 1$. Therefore, condition $\left(C_{2}\right)$ of Lemma 2.4 is satisfied. To check condition $\left(C_{1}\right)$ of Lemma 2.4 , we select $u(t)=\frac{b+c}{2}, 0 \leq$ $t \leq 1$. So, $u(t)=\frac{b+c}{2} \in P(\theta, b, c), \theta(u)=\theta\left(\frac{b+c}{2}\right)>b$, consequently, $\{u \in P(\theta, b, c): \theta(u)>$ $b\} \neq \phi$.

From assumption $\left(A_{2}\right)$, we have, $f(t, u) \geq \frac{\Gamma(\beta)}{\rho^{1-\beta}} \phi_{p}(M b)$, for $t \in[1 / 4,3 / 4]$. So,

$$
\begin{aligned}
\theta(T u) & =\min _{1 / 4 \leq t \leq 3 / 4}|T u(t)| \\
& \geq M b \frac{\rho^{1-\alpha}}{\Gamma(\alpha)} \int_{1 / 4}^{3 / 4} \gamma\left(1-s^{\rho}\right)^{1-\alpha} \phi_{q}\left(\frac{s^{\beta \rho}}{\beta \rho}\right) d s=b
\end{aligned}
$$

for all $u \in P(\theta, b, c)$.

Hence by Lemma 2.4 the BVP (3.1) has at least three positive solutions $u_{1}, u_{2}$ and $u_{3}$.

Define the cone $Q \subset E$ by $Q=\left\{u \in E: u(t) \geq 0, u(t) \geq k_{1}\|u\|\right\}$, where $k_{1}$ is given by $k_{1}=\min \{k(s)\}<1$.

Here we give results for the existence of positive solutions of problem (4.1) by assuming that $f(t, u)=k_{1} f_{1}(t) f_{2}(u)$.

We define the operator $F: E \rightarrow E$ by $F u(t)=\int_{0}^{1} G(t, s) \phi_{q}\left({ }^{\rho} I_{0^{+}}^{\beta} f(s, u(s))\right) d s$. Further, we assume that $f_{1}$ and $f_{2}$ are nonnegative and $k_{1}>0$. We denote

$$
f_{0}=\lim _{u \rightarrow 0^{+}} \frac{f_{2}(u)}{\phi_{p}(u)}, \quad f_{\infty}=\lim _{u \rightarrow+\infty} \frac{f_{2}(u)}{\phi_{p}(u)} .
$$

Lemma 5.4. Let $F: Q \rightarrow E$ be the operator defined by

$$
F u(t)=\int_{0}^{1} G(t, s) \phi_{q}\left({ }^{\rho} I_{0^{+}}^{\beta} f(s, u(s))\right) d s
$$

Then $F: Q \rightarrow Q$ is completely continuous.

Proof. In the view of continuity and nonnegativeness of the functions $G$ and $f, F: Q \rightarrow Q$ is continuous.

Let $\Omega \subset Q$ be bounded, then for all $u \in \Omega$ there exists a positive constant $M_{1}>0$ such 
that $\|u\| \leq M_{1}$. Let $L=\underset{t \in[0,1], u \in\left[0, M_{1}\right]}{|f(t, u)|}+1$, then for $u \in \Omega$, we have

$$
\begin{aligned}
|F u| & \leq \int_{0}^{1} G(t, s) \phi_{q}\left({ }^{\rho} I_{0^{+}}^{\beta} f(s, u(s))\right) d s \\
& \leq \frac{\rho^{1-\alpha}}{\Gamma(\alpha)} \int_{0}^{1}\left(1-s^{\rho}\right)^{\alpha-1} s^{\rho-1} \phi_{q}\left(\frac{\rho^{1-\beta}}{\Gamma(\beta)} \int_{0}^{s}\left(s^{\rho}-\tau^{\rho}\right)^{\beta-1} \tau \rho-1\right) f(\tau, u(\tau) d \tau) d s \\
& \leq \frac{\rho^{1-\alpha}}{\Gamma(\alpha)} \int_{0}^{1}\left(1-s^{\rho}\right)^{\alpha-1} s^{\rho-1} \phi_{q}\left(\frac{L s^{\beta \rho}}{\rho^{\beta} \Gamma(\beta+1)}\right) d s \\
& =\frac{\rho^{1-\alpha}}{\Gamma(\alpha)} \phi_{q}\left(\frac{L}{\rho^{\beta} \Gamma(\beta+1)}\right) \int_{0}^{1}\left(1-s^{\rho}\right)^{\alpha-1} s^{\rho-1} \phi_{q}\left(s^{\beta \rho}\right) d s .
\end{aligned}
$$

Hence $F(\Omega)$ is uniformly bounded.

For any $u \in \Omega, t \in[0,1]$ and $0 \leq t_{1} \leq t_{2} \leq 1$, we obtain

$$
\left|F u\left(t_{1}\right)-F u\left(t_{2}\right)\right|=\int_{0}^{1}\left|G\left(t_{1}, s\right)-G\left(t_{2}, s\right)\right| \phi_{q}\left({ }^{\rho} I_{0^{+}}^{\beta} f(s, u(s))\right) d s
$$

where

$$
\begin{aligned}
G\left(t_{1}, s\right)-G\left(t_{2}, s\right) & =\frac{\rho^{1-\alpha}}{\Gamma(\alpha)} s^{\rho-1}\left(t_{1}^{\rho(\alpha-1)}-t_{2}^{\rho(\alpha-1)}\right) \\
& \left\{\begin{array}{l}
\left.\left(1-s^{\rho}\right)^{\alpha-1}-\left(\left(t_{1}^{\rho}-s^{\rho}\right)^{\alpha-1}\right)-\left(t_{2}^{\rho}-s^{\rho}\right)^{\alpha-1}\right), \quad 0 \leq s \leq t \leq 1 \\
\left(1-s^{\rho}\right)^{\alpha-1}, \quad 0 \leq t \leq s \leq 1 .
\end{array}\right.
\end{aligned}
$$

Clearly, as $t_{1} \rightarrow t_{2}, \quad G\left(t_{1}, s\right)-G\left(t_{2}, s\right) \rightarrow 0$.

This implies that $F(\Omega)$ is equicontinuous. Hence by Arzela-Ascoli theorem $F: Q \rightarrow Q$ is completely continuous.

Theorem 5.5. Let (5.4) hold. Assume that if

$\left(H_{1}\right) f_{0}=\infty$ and $f_{\infty}=0$, or

$\left(H_{2}\right) f_{0}=0$ and $f_{\infty}=\infty$,

then for all $k_{1}>0$ the boundary value problem (4.1) has a positive solution.

Proof. Consider $\left(H_{1}\right)$. Since $f_{0}=\infty$, then there exists a constant $\rho_{1}>0$ such that $f_{2}(u)>\phi_{p}(M|u|)$ for $0<|u|<\rho_{1}$, where

$$
M>\left(\int_{1 / 4}^{3 / 4} k(s)\left(1-s^{\rho}\right)^{\alpha-1} s^{\rho-1} \phi_{q}\left(\frac{k_{1} \rho^{1-\beta}}{\Gamma(\beta)} \int_{0}^{s}\left(s^{\rho}-\tau^{\rho}\right)^{\beta-1} \tau^{\rho-1} f_{1}(\tau) d \tau\right) d s\right)^{-1} .
$$

Take $u \in Q$, such that $\|u\|=\rho_{1}$, then

$$
\begin{aligned}
\|F u\| & =\max _{t \in[0,1]}\left|\int_{0}^{1} G(t, s) \phi_{q}\left({ }^{\rho} I_{0^{+}}^{\beta} f(s, u(s))\right) d s\right| \\
& \geq \int_{1 / 4}^{3 / 4} k(s)\left(1-s^{\rho}\right)^{\alpha-1} s^{\rho-1} \phi_{q}\left(\frac{k_{1} \rho^{1-\beta}}{\Gamma(\beta)} \int_{0}^{s}\left(s^{\rho}-\tau^{\rho}\right)^{\beta-1} \tau^{\rho-1} f_{1}(\tau) \phi_{p}(M|u|) d \tau\right) d s \\
& \geq \int_{1 / 4}^{3 / 4} k(s)\left(1-s^{\rho}\right)^{\alpha-1} s^{\rho-1} \phi_{q}\left(\frac{k_{1} \rho^{1-\beta}}{\Gamma(\beta)} \int_{0}^{s}\left(s^{\rho}-\tau^{\rho}\right)^{\beta-1} \tau^{\rho-1} f_{1}(\tau) \phi_{p}\left(M k_{1}\|u\|\right) d \tau\right) d s \\
& \geq M\|u\| \int_{1 / 4}^{3 / 4} k(s)\left(1-s^{\rho}\right)^{\alpha-1} s^{\rho-1} \phi_{q}\left(\frac{k_{1} \rho^{1-\beta}}{\Gamma(\beta)} \int_{0}^{s}\left(s^{\rho}-\tau^{\rho}\right)^{\beta-1} \tau^{\rho-1} f_{1}(\tau) d \tau\right) d s \\
& >\|u\| .
\end{aligned}
$$

Now since $f_{\infty}=0$, there exists $N_{0}>0$ for

$$
N<\left(\frac{\rho^{1-\alpha}}{\Gamma(\alpha)} \int_{0}^{1}\left(1-s^{\rho}\right)^{\alpha-1} s^{\rho-1}|u| \phi_{q}\left(\frac{\rho^{1-\beta}}{\Gamma(\beta)} \int_{0}^{s}\left(s^{\rho}-\tau^{\rho}\right)^{\beta-1} \tau^{\rho-1} f_{1}(\tau) d \tau\right) d s\right)^{-1},
$$


such that $f_{2}(u) \leq \phi_{p}(N|u|)$ for $|u| \geq N_{0}$. Therefore, $f_{2}(u) \leq \phi_{p}(N|u|+\epsilon)$, where $\epsilon=$ $\max _{0 \leq u \leq N_{0}} f_{2}(u)+1$. Let $\Omega_{\rho_{2}}=\left\{u \in Q /\|u\|<\rho_{2}\right\}$. Take $u \in Q$ such that $\|u\|=\rho_{2}$ where $\rho_{2}>\left\{\rho_{1}, 2 \epsilon \frac{\rho^{1-\alpha}}{\Gamma(\alpha)} \int_{0}^{1}\left(1-s^{\rho}\right)^{\alpha-1} s^{\rho-1} \phi_{q}\left(\frac{\rho^{1-\beta}}{\Gamma(\beta)} \int_{0}^{s}\left(s^{\rho}-\tau^{\rho}\right)^{\beta-1} \tau^{\rho-1} f_{1}(\tau) d \tau\right) d s\right\}$, so, we have

$$
\begin{aligned}
|F u| \leq & \frac{\rho^{1-\alpha}}{\Gamma(\alpha)} \int_{0}^{1}\left(1-s^{\rho}\right)^{\alpha-1} s^{\rho-1} \phi_{q}\left(\frac{k_{1} \rho^{1-\beta}}{\Gamma(\beta)} \int_{0}^{s}\left(s^{\rho}-\tau^{\rho}\right)^{\beta-1} \tau^{\rho-1} f_{1}(\tau) \phi_{p}(N|u|+\epsilon) d \tau\right) d s \\
\leq & \frac{\rho^{1-\alpha}}{\Gamma(\alpha)} \int_{0}^{1}\left(1-s^{\rho}\right)^{\alpha-1} s^{\rho-1}(N|u|+\epsilon) \phi_{q}\left(\frac{\rho^{1-\beta}}{\Gamma(\beta)} \int_{0}^{s}\left(s^{\rho}-\tau^{\rho}\right)^{\beta-1} \tau^{\rho-1} f_{1}(\tau) d \tau\right) d s \\
\leq & \frac{\rho^{1-\alpha}}{\Gamma(\alpha)} N \int_{0}^{1}\left(1-s^{\rho}\right)^{\alpha-1} s^{\rho-1}|u| \phi_{q}\left(\frac{\rho^{1-\beta}}{\Gamma(\beta)} \int_{0}^{s}\left(s^{\rho}-\tau^{\rho}\right)^{\beta-1} \tau^{\rho-1} f_{1}(\tau) d \tau\right) d s \\
& +\frac{\rho^{1-\alpha}}{\Gamma(\alpha)} \epsilon \int_{0}^{1}\left(1-s^{\rho}\right)^{\alpha-1} s^{\rho-1} \phi_{q}\left(\frac{\rho^{1-\beta}}{\Gamma(\beta)} \int_{0}^{s}\left(s^{\rho}-\tau^{\rho}\right)^{\beta-1} \tau^{\rho-1} f_{1}(\tau) d \tau\right) d s \\
\leq & \frac{\rho_{2}}{2}+\frac{\rho_{2}}{2}=\rho_{2} .
\end{aligned}
$$

This implies $\|F u\| \leq\|u\|$. It follows from Lemma 2.5 (ii) that $F$ has at least one fixed point $u$ in $Q \cap\left(\bar{\Omega}_{\rho_{2}} / \Omega_{\rho_{1}}\right)$.

Now consider $\left(H_{2}\right)$, that is $f_{0}=0$ and $f_{\infty}=\infty$.

Now, since $f_{0}=0$, then there exists a positive constant $\rho_{1}$ such that $f_{2}(u)<\phi_{p}(\epsilon|u|)$, whenever $0<|u|<\rho_{1}$, where

$$
\left.\epsilon<\left(\frac{\rho^{1-\alpha}}{\Gamma(\alpha)} \int_{0}^{1}\left(1-s^{\rho}\right)^{\alpha-1} s^{\rho-1} \phi_{q}\left(\frac{k_{1} \rho^{1-\alpha}}{\Gamma(\beta)} \int_{0}^{s}\left(s^{\rho}-\tau^{\rho}\right)^{\beta-1}\right) \tau^{\rho-1} f_{1}(\tau) d \tau\right) d s\right)^{-1} .
$$

Let $\Omega_{\rho_{1}}=\left\{u \in Q:\|u\|<\rho_{1}\right\}$. For $u \in Q$, such that $\|u\|=\rho_{1}$, we have

$$
\begin{aligned}
|F u| & \leq\left|\frac{\rho^{1-\alpha}}{\Gamma(\alpha)} \int_{0}^{1}\left(1-s^{\rho}\right)^{\alpha-1} s^{\rho-1} \phi_{q}\left({ }^{\rho} I_{0^{+}}^{\beta} f(s, u(s))\right) d s\right| \\
& \leq \frac{\rho^{1-\alpha}}{\Gamma(\alpha)} \int_{0}^{1}\left(1-s^{\rho}\right)^{\alpha-1} s^{\rho-1} \phi_{q}\left(\frac{k_{1} \rho^{1-\beta}}{\Gamma(\beta)} \int_{0}^{s}\left(s^{\rho}-\tau^{\rho}\right)^{\beta-1} \tau^{\rho-1} f_{1}(\tau) \phi_{p}(\epsilon|u|) d \tau\right) d s \\
& \leq \frac{\rho^{1-\alpha}}{\Gamma(\alpha)} \int_{0}^{1}\left(1-s^{\rho}\right)^{\alpha-1} s^{\rho-1} \phi_{q}\left(\frac{k_{1} \rho^{1-\beta}}{\Gamma(\beta)} \int_{0}^{s}\left(s^{\rho}-\tau^{\rho}\right)^{\beta-1} \tau^{\rho-1} f_{1}(\tau) \phi_{p}(\epsilon\|u\|) d \tau\right) d s \\
& \leq \epsilon\|u\| \frac{\rho^{1-\alpha}}{\Gamma(\alpha)} \int_{0}^{1}\left(1-s^{\rho}\right)^{\alpha-1} s^{\rho-1} \phi_{q}\left(\frac{k_{1} \rho^{1-\beta}}{\Gamma(\beta)} \int_{0}^{s}\left(s^{\rho}-\tau^{\rho}\right)^{\beta-1} \tau^{\rho-1} f_{1}(\tau) d \tau\right) d s \\
& <\|u\|,
\end{aligned}
$$

which implies $\|F u\| \leq\|u\|$. Next, as $f_{\infty}=\infty$, there exists $N_{0}>0$ such that $f_{2}(u)>$ $\phi_{p}(M|u|)$, whenever $N_{0}<|u|$, where

$$
M>\left(\int_{1 / 4}^{3 / 4} k(s)\left(1-s^{\rho}\right)^{\alpha-1} s^{\rho-1} \phi_{q}\left(\frac{k_{1} \rho^{1-\beta}}{\Gamma(\beta)} \int_{0}^{s}\left(s^{\rho}-\tau^{\rho}\right)^{\beta-1} \tau^{\rho-1} f_{1}(\tau) d \tau\right) d s\right)^{-1} .
$$

Let $\Omega_{\rho_{2}}=\left\{u \in Q:\|u\|<\rho_{2}\right\}$. Take $u \in Q$ such that $\|u\|=\rho_{2}$, where $\rho_{2}>\left\{\rho_{1}, \frac{N_{0}}{k}\right\}$. For $1 / 4 \leq t \leq 3 / 4$, using the same procedure as above, we obtain $\|F u\|>\|u\|$.

Hence from Lemma 2.5, it follows that $F$ has at least one fixed point $u$ in $Q \cap\left(\bar{\Omega}_{\rho_{2}} / \Omega_{\rho_{1}}\right)$.

Define the cone $K \subset E$ by

$$
K=\{u \in E: u(t) \geq 0,0 \leq t \leq 1\} .
$$


Lemma 5.6. Let $\Phi: K \rightarrow E$ be the operator defined by

$$
\begin{aligned}
\Phi u(t)= & \int_{0}^{1} G(t, s) \phi_{q}\left({ }^{\rho} I_{0^{+}}^{\beta} f(s, u(s))\right) d s \\
= & -\frac{\rho^{1-\alpha}}{\Gamma(\alpha)} \int_{0}^{t}\left(t^{\rho}-s^{\rho}\right)^{\alpha-1} s^{\rho-1} \phi_{q}\left({ }^{\rho} I_{0^{+}}^{\beta} f(s, u(s))\right) d s \\
& +\frac{1}{\left(1-\beta \eta^{\rho(\alpha-1)}\right)} \frac{\rho^{1-\alpha}}{\Gamma(\alpha)} \int_{0}^{1} t^{\rho(\alpha-1)}\left(1-s^{\rho}\right)^{\alpha-1} s^{\rho-1} \phi_{q}\left({ }^{\rho} I_{0^{+}}^{\beta} f(s, u(s))\right) d s \\
& -\frac{\beta}{\left(1-\beta \eta^{\rho(\alpha-1)}\right)} \frac{\rho^{1-\alpha}}{\Gamma(\alpha)} \int_{0}^{\eta} t^{\rho(\alpha-1)}\left(\eta^{\rho}-s^{\rho}\right)^{\alpha-1} s^{\rho-1} \phi_{q}\left({ }^{\rho} I_{0^{+}}^{\beta} f(s, u(s))\right) d s,
\end{aligned}
$$

where $G(t, s)$ is defined as (4.7). Then $\Phi: K \rightarrow K$ is completely continuous.

Proof. As a result of the continuity and nonnegativity of $G(t, s)$ and $f(t, u)$, the operator $\Phi: K \rightarrow K$ is continuous. Let $\Omega \subset K$ be bounded, then there exists a constant $M>0$ such that $\|u\| \leq M$, for all $u \in \Omega$. Let $L=|f(t, u(t))|+1$, then for $u \in \Omega$, we have $t \in[0,1], u \in[0, M]$

$$
\begin{aligned}
|\Phi u(t)| & =\left|\int_{0}^{1} G(t, s) \phi_{q}\left({ }^{\rho} I_{0^{+}}^{\beta} f(s, u(s))\right) d s\right| \\
& \leq \phi_{q}\left(\frac{L}{\rho^{\beta} \Gamma(\beta+1)}\right) \int_{0}^{1} \max _{0 \leq t \leq 1} G(t, s) \phi_{q}\left(s^{\beta \rho}\right) d s .
\end{aligned}
$$

Hence, $\Phi(\Omega)$ is bounded. For each $u \in \Omega, t_{1}, t_{2} \in[0,1], t_{1}<t_{2}$, we have

$$
\begin{aligned}
\mid \Phi u\left(t_{2}\right)- & \Phi u\left(t_{1}\right)|=|-\frac{\rho^{1-\alpha}}{\Gamma(\alpha)} \int_{0}^{t}\left(t^{\rho}-s^{\rho}\right)^{\alpha-1} s^{\rho-1} \phi_{q}\left({ }^{\rho} I_{0^{+}}^{\beta} f(s, u(s))\right) d s \\
& +\frac{1}{\left(1-\beta \eta^{\rho(\alpha-1)}\right)} \frac{\rho^{1-\alpha}}{\Gamma(\alpha)} \int_{0}^{1} t^{\rho(\alpha-1)}\left(1-s^{\rho}\right)^{\alpha-1} s^{\rho-1} \phi_{q}\left({ }^{\rho} I_{0^{+}}^{\beta} f(s, u(s))\right) d s \\
& -\frac{\beta}{\left(1-\beta \eta^{\rho(\alpha-1)}\right)} \frac{\rho^{1-\alpha}}{\Gamma(\alpha)} \int_{0}^{\eta} t^{\rho(\alpha-1)}\left(\eta^{\rho}-s^{\rho}\right)^{\alpha-1} s^{\rho-1} \phi_{q}\left({ }^{\rho} I_{0^{+}}^{\beta} f(s, u(s))\right) d s \mid \\
\leq & \mid \frac{\rho^{1-\alpha}}{\Gamma(\alpha)} \int_{0}^{t_{2}}\left(t_{2}^{\rho}-s^{\rho}\right)^{\alpha-1} s^{\rho-1} \phi_{q}\left({ }^{\rho} I_{0^{+}}^{\beta} f(s, u(s))\right) d s \\
& -\frac{\rho^{1-\alpha}}{\Gamma(\alpha)} \int_{0}^{t_{1}}\left(t_{1}^{\rho}-s^{\rho}\right)^{\alpha-1} s^{\rho-1} \phi_{q}\left({ }^{\rho} I_{0^{+}}^{\beta} f(s, u(s))\right) d s \mid \\
& +\mid \frac{1}{\left(1-\beta \eta^{\rho(\alpha-1)}\right)} \frac{\rho^{1-\alpha}}{\Gamma(\alpha)} \int_{0}^{1} t_{2}^{\rho(\alpha-1)}\left(1-s^{\rho}\right)^{\alpha-1} s^{\rho-1} \phi_{q}\left({ }^{\rho} I_{0^{+}}^{\beta} f(s, u(s))\right) d s \\
& -\frac{1}{\left(1-\beta \eta^{\rho(\alpha-1)}\right)} \frac{\rho^{1-\alpha}}{\Gamma(\alpha)} \int_{0}^{1} t_{1}^{\rho(\alpha-1)}\left(1-s^{\rho}\right)^{\alpha-1} s^{\rho-1} \phi_{q}\left({ }^{\rho} I_{0^{+}}^{\beta} f(s, u(s)) \mid\right. \\
& +\mid \frac{\beta}{\left(1-\beta \eta^{\rho(\alpha-1)}\right)} \frac{\rho^{1-\alpha}}{\Gamma(\alpha)} \int_{0}^{\eta} t_{2}^{\rho(\alpha-1)}\left(\eta^{\rho}-s^{\rho}\right)^{\alpha-1} s^{\rho-1} \phi_{q}\left({ }^{\rho} I_{0^{+}}^{\beta} f(s, u(s))\right) d s \\
& -\frac{\beta}{\left(1-\beta \eta^{\rho(\alpha-1)}\right)} \frac{\rho^{1-\alpha}}{\Gamma(\alpha)} \int_{0}^{\eta} t_{1}^{\rho(\alpha-1)}\left(\eta^{\rho}-s^{\rho}\right)^{\alpha-1} s^{\rho-1} \phi_{q}\left({ }^{\rho} I_{0^{+}}^{\beta} f(s, u(s))\right) d s \mid \\
\leq & \frac{\rho^{1-\alpha}}{\Gamma(\alpha)} \phi_{q}\left(\frac{L}{\rho^{\beta} \Gamma(\beta+1)}\right) \mid \int_{0}^{t_{1}}\left(\left(t_{2}^{\rho}-s^{\rho}\right)^{\alpha-1}-\left(t_{1}^{\rho}-s^{\rho}\right)^{\alpha-1}\right) s^{\rho-1} \phi_{q}\left(s^{\beta \rho}\right) d s \\
& +\int_{t_{1}}^{t_{2}}\left(t_{2}^{\rho}-s^{\rho}\right)^{\alpha-1} s^{\rho-1} \phi_{q}\left(s^{\beta \rho}\right) d s \mid \\
& +\frac{\left|t_{2}^{\rho(\alpha-1)}-t_{1}^{\rho(\alpha-1)}\right|}{\left(1-\beta \eta^{\alpha-1}\right)} \phi_{q}\left(\frac{L}{\rho^{\beta} \Gamma(\beta+1)}\right) \int_{0}^{1}\left(1-s^{\rho}\right)^{\alpha-1} s^{\rho-1} \phi_{q}\left(s^{\beta \rho}\right) d s \\
&
\end{aligned}
$$




$$
\begin{aligned}
& +\frac{\beta\left|t_{2}^{\rho(\alpha-1)}-t_{1}^{\rho(\alpha-1)}\right|}{\left(1-\beta \eta^{\alpha-1}\right)} \phi_{q}\left(\frac{L}{\rho^{\beta} \Gamma(\beta+1)}\right) \int_{0}^{\eta}\left(\eta^{\rho}-s^{\rho}\right)^{\alpha-1} s^{\rho-1} \phi_{q}\left(s^{\beta \rho}\right) d s \\
\leq & \frac{\rho^{1-\alpha}}{\Gamma(\alpha)} \phi_{q}\left(\frac{L}{\rho^{\beta} \Gamma(\beta+1)}\right)\left(t_{2}-t_{1}\right)\left|\int_{0}^{t_{1}} h^{\prime}(\xi) s^{\rho-1} \phi_{q}\left(s^{\beta \rho}\right) d s\right| \\
& +\left|\int_{t_{1}}^{t_{2}}\left(t_{2}^{\rho}-s^{\rho}\right)^{\alpha-1} s^{\rho-1} \phi_{q}\left(s^{\beta \rho}\right) d s\right| \\
& +\frac{\left|t_{2}^{\rho(\alpha-1)}-t_{1}^{\rho(\alpha-1)}\right|}{\left(1-\beta \eta^{\alpha-1}\right)} \phi_{q}\left(\frac{L}{\rho^{\beta} \Gamma(\beta+1)}\right) \int_{0}^{1}\left(1-s^{\rho}\right)^{\alpha-1} s^{\rho-1} \phi_{q}\left(s^{\beta \rho}\right) d s \\
& +\frac{\beta\left|t_{2}^{\rho(\alpha-1)}-t_{1}^{\rho(\alpha-1)}\right|}{\left(1-\beta \eta^{\alpha-1}\right)} \phi_{q}\left(\frac{L}{\rho^{\beta} \Gamma(\beta+1)}\right) \int_{0}^{\eta}\left(\eta^{\rho}-s^{\rho}\right)^{\alpha-1} s^{\rho-1} \phi_{q}\left(s^{\beta \rho}\right) d s \rightarrow 0,
\end{aligned}
$$

as $t_{1} \rightarrow t_{2}$. Thus, by means of Arzela-Ascoli theorem, $\Phi: K \rightarrow K$ is completely continuous.

Theorem 5.7. Suppose $f(t, u)$ satisfies $f(t, 0) \neq 0, t \in(0,1)$ and

$$
0 \leq \varlimsup_{u \rightarrow \infty} \max _{t \in[0,1]} \frac{f(t, u)}{\phi_{p}(u)}<\left(1-\beta \eta^{\rho(\alpha-1)}\right) \Gamma(\alpha) .
$$

Then, the problem (4.6) has at least one positive solution.

Proof. In view of the continuity and nonnegativity of $f$ from (5.5), there exists $\lambda>0,0<$ $M<\left(1-\beta \eta^{\rho(\alpha-1)}\right) \Gamma(\alpha)$ such that for $t \in[0,1], u \in[0,+\infty)$, we have

$$
0 \leq f(t, u)<\phi_{p}\left(\frac{M}{\rho^{\beta} \Gamma(\beta+1)} u+\lambda\right) .
$$

Let

$$
K_{R}=\left\{u \in K:\left\|u-\lambda \int_{0}^{1} G(t, s) \phi_{q}\left(s^{\beta \rho}\right) d s\right\| \leq R\right\}
$$

be a convex, bounded, and closed subset of the Banach space $E$.

For $u \in K_{R}$, we obtain

$$
\begin{aligned}
\|u\| & \leq \lambda\left\|\int_{0}^{1} G(t, s) \phi_{q}\left(s^{\beta \rho}\right) d s\right\|+R \\
& \leq R+\frac{\lambda}{\left(1-\beta \eta^{\rho(\alpha-1)}\right) \Gamma(\alpha)},
\end{aligned}
$$

and

$$
\begin{aligned}
\left|\Phi u(t)-\lambda \int_{0}^{1} G(t, s) \phi_{q}\left(s^{\beta \rho}\right) d s\right| & \leq \int_{0}^{1} G(t, s)\left|\phi_{q}\left({ }^{\rho} I_{0^{+}}^{\beta} f(s, u(s))-\lambda \phi_{q}\left(s^{\beta \rho}\right)\right)\right| d s \\
& \leq \max \left\{\frac{M\|u\|}{\left(1-\beta \eta^{\rho(\alpha-1)}\right) \Gamma(\alpha)}, \frac{\lambda}{\left(1-\beta \eta^{\rho(\alpha-1)}\right) \Gamma(\alpha)}\right\} \\
& \leq \max \left\{\frac{M\left[R+\frac{\lambda}{\left(1-\beta \eta^{\rho(\alpha-1)}\right) \Gamma(\alpha)}\right]}{\left(1-\beta \eta^{\rho(\alpha-1)}\right) \Gamma(\alpha)}, \frac{\lambda}{\left(1-\beta \eta^{\rho(\alpha-1)}\right) \Gamma(\alpha)}\right\} \\
& \leq R,
\end{aligned}
$$

whenever $R \geq \frac{\lambda}{\left(1-\beta \eta^{\rho(\alpha-1)}\right) \Gamma(\alpha)-M} \frac{M}{\left(1-\beta \eta^{\rho(\alpha-1)}\right) \Gamma(\alpha)}$.

Hence by Schauder fixed point theorem the operator $\Phi$ has at least one fixed point in $K_{R}$. 
Example 5.8. Consider the boundary value problem of generalized fractional differential equation

$$
\begin{gathered}
{ }_{c}^{\rho} D_{0^{+}}^{\beta}\left(\phi_{p}\left({ }_{c}^{\rho} D_{0^{+}}^{\alpha} u(t)\right)\right)+f(t, u(t))=0, \quad t \in(0,1), \\
u(0)=u(1)=u^{\prime \prime}(0)=0,{ }_{c}^{\rho} D_{0^{+}}^{\alpha} u(0)=0,
\end{gathered}
$$

where $\rho=3 / 2, \beta=1 / 2, p=4 / 3, \alpha=5 / 2$ and $f(t, u(t))=\sqrt{t}+\frac{|\sin u|}{1+t^{3}}$.

By computations, we have

$$
\begin{aligned}
& N=\frac{\Gamma(\alpha)}{\rho^{1-\alpha}}\left(\int_{0}^{1}\left(1-s^{\rho}\right)^{\alpha-1} \phi_{q}\left(\frac{s^{\beta \rho}}{\beta \rho}\right) d s\right)^{-1}=5, \\
& M=\frac{\Gamma(\alpha)}{\gamma \rho^{1-\alpha}}\left(\int_{1 / 4}^{3 / 4}\left(1-s^{\rho}\right)^{\alpha-1} \phi_{q}\left(\frac{s^{\beta \rho}}{\beta \rho}\right) d s\right)^{-1}=98 .
\end{aligned}
$$

We choose $\rho_{1}=1.5708, \rho_{2}=8$, thus we obtain

$$
\frac{\Gamma(\beta)}{\rho^{1-\beta}} \phi_{p}\left(\rho_{1} N\right) \approx 2.9, \frac{\Gamma(\beta)}{\rho^{1-\beta}} \phi_{p}\left(\rho_{2} M\right) \approx 13.34 .
$$

Now, for $(t, u) \in[0,1] \times\left[0, \rho_{1}\right], f(t, u(t)) \leq 1.59899$ and for $(t, u) \in[1 / 4,3 / 4] \times\left[0, \rho_{2}\right]$, $f(t, u(t)) \geq 0.5$.

Conditions $\left(H_{1}\right)$ and $\left(H_{2}\right)$ of Theorem 5.2 hold, therefore, the problem (5.6) has at least one positive solution $u$ such that $1.5708 \leq\|u\| \leq 8$.

Example 5.9. Consider the boundary value problem of the generalized fractional differential equation

$$
\begin{array}{r}
{ }^{\rho} D_{0^{+}}^{\beta}\left(\phi_{p}\left({ }^{\rho} D_{0^{+}}^{\alpha} u(t)\right)\right)+f(t, u(t))=0, \quad t \in(0,1), \\
u(0)=u(1)=0,{ }^{\rho} D_{0^{+}}^{\alpha} u(0)=0,
\end{array}
$$

where $\beta=1 / 2, \rho=2, p=2, \alpha=3 / 2$, and $f(t, u(t))=k_{1} \frac{u^{3} t^{2}}{2} e^{\frac{u \pi}{2}}$, where $f_{1}(t)=$ $\frac{t^{2}}{2}, f_{2}(u)=u^{3} e^{\frac{\pi u}{2}}$ satisfy (5.4) and the condition $\left(H_{2}\right)$ of Theorem 5.5. For $k=3 / 5$, we have $k_{1}=1 / 4>0$. Therefore, the boundary value problem (5.7) has a positive solution.

\section{References}

[1] B. Ahmad, M. Alghanmi, S.K. Ntouyas and A. Alsaedi, Fractional differential equations involving generalized derivative with Stieltjes and fractional integral boundary conditions, Appl. Math. Lett. 84, 111-117, 2018.

[2] A. Ali, F. Rabiei and K. Shah, On Ulam's type stability for a class of impulsive fractional differential equations with nonlinear integral boundary conditions, J. Nonlinear. Sci. Appl. 10, 4760-4775, 2017.

[3] A. Ali, K. Shah and D. Baleanu, Ulam stability results to a class of nonlinear implicit boundary value problems of impulsive fractional differential equations, Adv. Differ. Equ. 5, 1-21, 2019.

[4] R. Almeida, A.B. Malinowska and M.T.T. Monteiro, Fractional differential equations with a Caputo derivative with respect to a Kernel function and their applications, Math. Method. Appl. Sci. 41, 336-352, 2018.

[5] Asma, A. Ali, K. Shah and F. Jarad, Ulam-Hyers stability analysis to a class of nonlinear implicit impulsive fractional differential equations with three point boundary conditions, Adv. Differ. Equ. 7, 1-27, 2019.

[6] Z. Bai, On positive solutions of a nonlocal fractional boundary value problem, Nonlinear Anal. 72, 916-924, 2010. 
[7] Z. Bai and H. Lu, Positive solutions for boundary value problem of nonlinear fractional differential equation, J. Math. Anal. Appl. 311, 495-505, 2005.

[8] A. Benlabbes, M. Benbachir and M. Lakrib, Boundary value problems for nonlinear fractional differential equations, Facta. Univ. Ser. Math. Inform. 30 (2), 157-168, 2015.

[9] A. Cabada and G. Wang, Positive solutions of nonlinear fractional differential equations with integral boundary value conditions, J. Math. Anal. Appl. 389, 403-411, 2012.

[10] T. Chen, W. Liu and Z. Hu, A boundary value problem for fractional differential equation with p-Laplacian operator at resonance, Nonlinear Anal. 75, 3210-3217, 2012.

[11] W. Chen and Y. Zhao, Solvability of boundary value problems of nonlinear fractional differential equations, Adv. Differ. Equ., Art. No. 36, 2015.

[12] Y. Cui, S. Kang and Z. Liu, Existence of positive solutions to boundary value problem of Caputo fractional differential equation, Discrete Dyn. Nat. Soc., Art. ID 708053, 6 pp., 2015.

[13] X. Dong, Z. Bai and S. Zhang, Positive solutions to boundary value problems of pLaplacian with fractional derivative, Bound. Value. Probl., Art. No. 5, 2017.

[14] D. Guo and V. Lakshmikantham, Nonlinear Problems in Abstract Cones, Academic Press, Boston, 1988.

[15] F. Jarad, T. Abdeljawad and D. Baleanu, On the generalized fractional derivatives and their Caputo modification, J. Nonlinear Sci. Appl. 10, 2607-2619, 2017.

[16] D. Jiang and C. Yuan, The positive properties of the Green function for Dirichlettype boundary value problems of nonlinear fractional differential equations and its application, Nonlinear Anal. 72, 710-719, 2010.

[17] U.N. Katugampola, New approach to a generalized fractional integral, Appl. Math. Comput. 218, 860-865, 2011.

[18] U.N. Katugampola, A new approach to generalized fractional derivatives, Bull. Math. Anal. App. 6 (4), 1-15, 2014.

[19] U.N. Katugampola, Existence and uniqueness results for a class of generalized fractional differential equations, arXiv: $1411.5229 \mathrm{v} 2[$ math.CA].

[20] Y. Li and G. Li, Positive solutions of p-Laplacian fractional differential equations with integral boundary value conditions, J. Nonlinear Sci. Appl. 9, 717-726, 2016.

[21] Y. Li, K. Shah and R.A. Khan, Iterative technique for coupled integral boundary value problem of non-linear of non-integer order differential equations, Adv. Differ. Equ. 251, 2017.

[22] S. Liang and J. Zhang, Positive solutions for boundary value problems of nonlinear fractional differential equation, Nonlinear Anal. 71, 5545-5550, 2009.

[23] K. Shah, A. Ali and S. Bushnaq, Hyers-Ulam stability analysis to implicit Cauchy problem of fractional differential equations with impulsive condition, Math. Method. Appl. Sci. 41 (17), 8329-8343, 2018.

[24] K. Shah, H. Khalil and R.A. Khan, Upper and lower solutions to a coupled system of nonlinear fractional differential equations, Progr. Fract. Differ. Appl. 2 (1), 1-10, 2016.

[25] K. Shah, R.A. Khan, Study of solution to a toppled system of fractional differential equations with integral boundary conditions, Int. J. Appl. Comput. Math. 3 (3), 2369$2388,2017$.

[26] X. Su and S. Zhang, Solutions to boundary value problems for nonlinear differential equations of fractional order, Electron. J. Differ. Eq. 26, 1-15, 2009.

[27] Y. Sun, Positive solutions for third-order three-point nonhomogeneous boundary value problems, Appl. Math. Lett. 22, 45-51, 2009. 
[28] J. Tan and M. Li, Solutions of fractional differential equations with p-Laplacian operator in Banach spaces, Bound. Value. Probl., Art. No. 15, 2018.

[29] Y. Wang and L. Liu, Positive properties of the Green function for two-term fractional differential equations and its application, J. Nonlinear Sci. Appl. 10, 2094-2102, 2017.

[30] L. Yang, X.P. Liu and M. Jia, Multiplicity results for second order m-point boundary value problem, J. Math. Anal. Appl. 324 (1), 532-542, 2006.

[31] S. Zhang, Positive solutions for boundary value problems of nonlinear fractional differential equations, Electron. J. Differ. Eq. 36, 1-12, 2006.

[32] S. Zhang, Positive solution of singular boundary value problem for nonlinear fractional differential equation with nonlinearity that changes sign, Positivity 16, 177-193, 2012. 